\title{
Beyond Wearables: Experiences and Trends in Design of Portable Medical Devices
}

\author{
Rafael Gomez and Anna Harrison \\ Queensland University of Technology, School of Design, \\ Creative Industries Faculty, Australia \\ (r.gomez, anna.harrison) @qut.edu.au
}

\begin{abstract}
The use of Portable Medical Devices (PMDs) has become increasingly widespread over the last few years. A combination of factors; including advances in technology, the pressure to reduce public health costs and the desire to make health solutions accessible to a wider patient base are contributing to the growth in the PMD market. Design has a clear role to play in the current and future context of the PMD landscape. In this paper, we identify emerging trends in the design of PMDs; including changes in the form, purpose and mode of use, and explore how these trends are likely to fundamentally impact the nature of healthcare and the patient experience from an experience design perspective. We conclude by identifying a research opportunity for design within the healthcare and PMD context.
\end{abstract}

Keywords: Portable Medical Device, Implantable Medical Devices, Mobile Health, Patient Experience, Experience Design.

\section{Introduction}

Portable medical devices (PMDs) have become commonplace for many people around the world, and this trend is likely to continue. These devices impact the ways in which people monitor, manage, maintain, regulate and ultimately determine their own health and wellbeing. PMDs play a critical role in health management that can lead to reduced pressure on the healthcare system, curtail unhealthy habits, improve adherence to medical regimes, increase likelihood of improved lifestyle choices and enhance the ongoing experience of health management $[1,2]$. With the rise of chronic health conditions [3] innovations in this area have the potential to improve and enhance the experience and overall quality of life for patients. PMDs not only allow ongoing, continuous, and unobtrusive monitoring of physiological patient conditions they can also provide more realistic indication of health status and information that is otherwise inaccessible through other means [4]. As PMD technology progresses we are seeing devices shift from operating externally to our bodies to being on our bodies as wearable devices and ultimately merge internally as implantable technologies. At that point the patient experience transforms and we enter an era where products themselves have the power to change not only the patient experience but also ultimately 
the human condition [5]. Implantable medical devices will have the capacity to rewire the human brain, and thus have a substantial impact on physiology, behavior, and emotions and people's overall health and wellbeing - radically changing the nature of healthcare and patient experience. Within the context of this paper PMDs are defined as small, portable (mobile, untethered) wireless computing devices to meet healthcare needs $[2,6]$. Due to their nature, PMDs enable people to be acutely aware of and monitor their health and wellbeing virtually at any time in any given context. From an experience design perspective, PMDs can be considered as mediators between the user and an external motivation to achieve a positive health outcome. They become a hinge by which patients can achieve a health related goal and permit or prohibit these goals from happening. But as we will discuss, when PMDs move beyond wearable to internal this dynamic changes and the interaction shifts from a conscious tangible experience to an unconscious intangible experience. As technology and other drivers continue to push the boundaries further an important question arises; how will trends in PMDs impact the nature of the patient and health experience? This presents a research opportunity for design, understanding and unpacking this new landscape is a step towards a dialogue that will lead to effective, and appropriate, design strategies for PMDs and healthcare experience. This paper discusses trends that have emerged beyond the current PMD landscape. Through this analysis existing PMDs are categorized along three dimensions, namely; form (dedicated or add-on), purpose (monitor, nudge, advanced diagnostic) and mode of use (external, wearable, internal), with mode of use being a driving factor for significant change. Future vision and directions are proposed and consideration of the potential impacts on the nature of healthcare and patient experience are discussed through the lens of experience design. By better understanding PMDs and impacts of future trends from an experience design perspective; researchers, designers and technology experts can contribute in a more meaningful way to the development of devices that ultimately raise the quality of life for patients now and in the future.

\section{The Rise of Portable Medical Devices}

It is evidently clear that the proliferation of 'anywhere, anytime' computing [7] has well and truly begun, and its inevitable path toward the medical and health field is already occurring. Devices will no longer just be portable they will soon be wearable and integrated into our bodies. In the not too distant future we will be experiencing medical devices that are embedded into our bodies to track, manage, diagnose and respond automatically and without our knowledge to our physical and physiological conditions. The convergence of the seemingly never ceasing advancement of smaller and more powerful computing with internet capabilities have created an environment in which patients can begin to be more active in their own personal medical and health management through PMDs. Innovations in technology are transforming the health care industry and spurring the development of portable medical devices for health management, tracking, monitoring, diagnosis, treatment and measuring of various health related data [2]. Health systems in many countries around the world are moving towards electronic healthcare systems (e-health) that automatically store 
personal, sensitive and medical information. These technical advances and developments are creating significant challenges for the industry [8,9] but also generate many opportunities in healthcare. Reducing public healthcare costs is another driving factor. Spiraling costs continue to be a challenging dilemma worldwide and with an ageing population the demand for healthcare will not dissipate anytime soon. In the United States healthcare costs account for $18 \%$ of GDP [2] and in Europe the same scenario prevails $[10,11]$. The current and emerging PMD technologies have the potential to reduce the strain on healthcare spending through better and faster decision-making processes, improve prevention strategies, reduce hospital visits and administrative costs, and improve adherence to treatment plans [2]. The desire to provide healthcare to a wider patient base is a further driver in this area [12]. Current healthcare system is founded on large-scale, capital intensive, centralized networks that demands individuals travel to hospitals and medical facilities. This system has become highly inefficient and PMDs can play a key role in transforming healthcare into a more efficient, patient-centered system. They permit individuals to have instant, on-demand access to their medical needs, health data and powerful clinical decision support tools that empower them to actively participate in their treatment plans. Portable devices also allow people to overcome the limitations of geography in rural areas, people in lower socioeconomic settings, people who suffer from mobility difficulties and cultural minorities [2,12,13]. These factors have been driving the advancement of PMDs at an everincreasing rate. PMDs are not only permeating modern life but will continue to do so for decades to come. The opportunities afforded are encouraging but it is essential to contextualize this within a design framework that situates the patient at the center in order to prepare for the shift in the nature of healthcare and ultimately provide an appropriate and positive patient experience.

\section{$3 \quad$ Experience and Design}

For over a decade, the field of design has seen an emerging interest in what has been termed as experience design. Experience design attempts to move past usability, functionality and effectiveness towards a more comprehensive understanding of the overall human-product interaction [14,15,16]. As [17] argues: "Experience design asserts design not to be about products anymore but about the experiences they deliver... meaning and emotion (becoming) the prime design objective " [17 p.75]. Users of products in everyday life, although concerned about practical and pragmatic issues, care more about about how products enhance and mediate daily experiences. So when talking about experiences this encompasses: emotions, interactions and user-productcontext relationship over time $[17,18,19,20]$. Experience design is relevant for PMDs as emotional experiences have a significant role to play in how people feel about themselves and about their health and can promote as well as hinder personal health [21]. [22] argues that well designed medical products lead to increased patience adherence to medical and take on a crucial role in patient therapy. Even more relevant perhaps is that if device design does not take into account patient experience this can lead to not only poor health regimes but also reduce people's quality of life and even 
lead to dangerous medical situations [21]. Within available research there seems to be minimal consideration of dealing with the emotional experience of interacting specifically with PMDs, especially when it comes to healthcare and the patient experience [23]. If the experience of interaction is not taken into account a gap in understanding the full spectrum of the patient experience can arise, as a result leading to inadequately designed services and devices. Research conducted by one of the authors on the emotional experience of portable interactive health and medical devices is one exception [23]. [24] describe two cases in which limited consideration of user experience leads to dangerous and hazardous results. Both cases involved the use of a defibrillator in a real world situation in which the design led to incorrect use of the device. In the first situation a medical team working on a cardiac arrest patient inadvertently delivered an electric shock leaving the patient with profound neurological injuries. The second case was a simulation where physicians were observed powering down a defibrillator when they intended to deliver a shock instead. Both cases appear like simple design flaws but also reveal a deeper issue about the experience. The authors argue that these devices failed because they were not designed to match the needs, cognitive processes, and environments of users [24]. To go one step further it is important to consider the experiential and emotional aspects of the users. For example in both cases stress, anxiety and concern are experiential aspects that can also contribute to incorrect use and errors with the device. Another paper published more recently discusses the importance of applying human factors approach to the medical device field [25]. The paper does a good job of stressing the relevance of including a human centered design approach. The authors go on to suggest the impact of the context of the experience as an important element of the relationship between user and product that is currently missing in studies of medical device design. Although [25] make a concerted effort to try and raise the level of design in the medical device industry by incorporating usability principles, if we are to truly acknowledge the complexity of user interactions with medical devices a broader experience design approach is needed. To better understand and design for the patient experience it is important to consider the latest in design research and knowledge. The experience design approach offers a perspective that encapsulates broader issues of experience design that form a part of the complex user-product relationship. Current and future PMDs should be designed appropriately to support the quality of life of people as they are implicated in influencing the way patients relate to their own health and wellbeing and can facilitate or inhibit positive experiences.

\section{Trends in the Portable Medical Device Landscape}

As PMDs continue to flourish there are some trends that are becoming evident. Instead of classifying these trends purely on technical capabilities the approach taken here is to view them along three interconnected dimensions, namely: form (dedicated or add-on), purpose (monitor, nudge and advanced diagnostic) and mode of use (external, wearable, internal). By looking at the existing literature from this novel perspective, several trends emerging in the PMD landscape can be observed and particular 
aspects in relation to the potential impact on the nature of healthcare and the patient experience can be identified.

Form. The first observed trend is related to the change in form of PMDs [26]. Here the form of health related technologies is broken into two types; dedicated devices for specific medical needs and add-ons to existing technologies that permit users to perform medical related functions that would otherwise not be able to be performed with a single device. Ever since the pacemaker, first used in 1959 [9] there have been a multitude of dedicated portable medical devices. More recently a multitude of manufacturers are developing PMDs for various needs such as diabetes and heart disease through to health and fitness devices for everyday use [2,12,27]. This area of the medical field is evolving rapidly but is also being absorbed in some cases by the development of add-on technologies. Health related add-ons are becoming more and more prevalent. One type that is growing rapidly is health related apps on mobile devices. There are apps that target almost any conceivable area of the health area including chronic conditions, telemedicine, remote monitoring, data capture, electronic records, e-prescribing, and health and fitness [2]. Services like WebMD are another form of add-on that have become available to patients on mobile devices, making selfdiagnosis easily accessible to the billions of people around the world who now carry a mobile phone. Peripheral products that attach to existing mobile devices and provide functionality not possible with the original device alone are another type of add-on technology. Example include a peripheral that attaches to a mobile phone for blood glucose monitoring and an eye testing device that attaches to a mobile phone camera [28,29].

Purpose. As PMDs have increased in variety so have the ways in which they are used. The second trend is classified according to the purpose that PMDs serve including monitoring, nudging and advanced diagnostics. PMDs used for monitoring medical information have been around for some time with electrocardiogram (ECG) and electroencephalogram (EEG) being some of the first portable devices used [30]. The AMON (advanced care and alert portable telemedical monitor) consists of a monitoring and alert system for high-risk cardiac and respiratory patients [31]. Recently monitoring functions have moved into the health and fitness area and can log data about people's exercise regime, habits and patterns with the Fitbit range of products and the Jawbone fitness tracker being examples of popular devices in this area. Beyond monitoring and data logging, PMDs have moved into what has been referred to as 'nudging' or persuasive functions [32]. A nudge refers to the ways in which a device motivates or prompts users to perform activities that improve the life of the individual and encourage them away from potentially harmful choices and nudge them towards healthy decisions. For instance, UbiFit transforms the background wallpaper on a mobile phone into a garden. Physical movement keeps the garden alive, thus encouraging users to be physically active and keep the garden nourished [33]. Another example is the Vito television remote control that offers viewers alternatives to watching TV including viewing "to do" lists and playing physical games [32]. A third group of PMDs are defined as advanced diagnostic devices. These devices support complex 
healthcare applications and enable continuous provision of health and medical needs by monitoring the patient, environment and context and respond accordingly. Some of the vitals signs these devices can monitor include electromyogram (EMG), (ECG), activity, mobility, falls, respiratory rate, heart sounds, blood glucose levels, oxygen saturation, body or skin temperature and galvanic skin response [34]. The Cardionet wearable system monitors patient heart rate, ECG and other data to help physicians diagnose and support patients suffering from arrhythmias [34]. [35] outline recent developments in ingestible medical devices that offer non-invasive alternatives to traditional endoscopy or surgery for diagnosis and treatment of gastrointestinal disorders.

Mode of Use. The ways in which PMDs are utilised have changed over the years from devices that are external to ones that are worn on the body to more recent devices that exist internally. Each change in mode has moved the device closer to the patient from fixed medical systems to mobile, wearable and ultimately implantable devices. The initial wave of PMDs were external to the body (with a few exceptions). As microelectronics and textile technology evolved wearable computing became common. Wearable computing is similar to portable or mobile devices but the term wearable suggests that the support environment is either the human body or a piece of clothing [27]. In this way wearable devices are closer in proximity to the body and have a different relationship to the person. Current developments in this area include implantable devices, which significantly alter the relationship of patient and device. The technology required for more modern implantable devices is also changing and impacting the user experience at a fundamental level as these devices, working at the nanoscale in some cases [36], are used for a variety of therapeutic or life-saving functions ranging from drug infusion and cardiac pacing to direct neurostimulation. Implantable devices can automatically and directly alter a patient physically, physiologically and in many cases without any patient interaction [8]. It is this third dimension that is interesting to discuss in terms of the health experience. This presents designers with a unique challenge because as devices move from external to wearable to internal, the ways in which we relate and interact with devices changes significantly. Implantable devices have the capacity to modify the patient's condition without the patient even knowing. This is a distinct change in user-product relationship that requires a new way of defining and evaluating the health experience. Form changes and transformations in purpose are relevant to outline but it is the change in mode of use that will perhaps have the biggest impact on the nature of healthcare and the patient experience. As dedicated and add-on devices begin to be implanted in our bodies performing monitoring, nudging and advanced diagnostic functions without patient interaction or awareness, it is crucial to begin to explore opportunities and question the role that design plays in this scenario.

\section{Future Visions for Potable Medical Devices}

As we move from the current state to a future vision some radical changes in the ways that patients experience PMDs can be envisaged. We have identified three dimensions 
that have recently evolved and following these trajectories we discuss the ways in which the next generation of PMDs impact the nature of healthcare and the patient experience.

Impact on Nature of Healthcare. Figure 1 outlines changes in the nature of healthcare in the future [2]. The current state of patient needs involves fitness, healthy living and wellbeing with a focus on home and remote monitoring and active access of health information by patients.

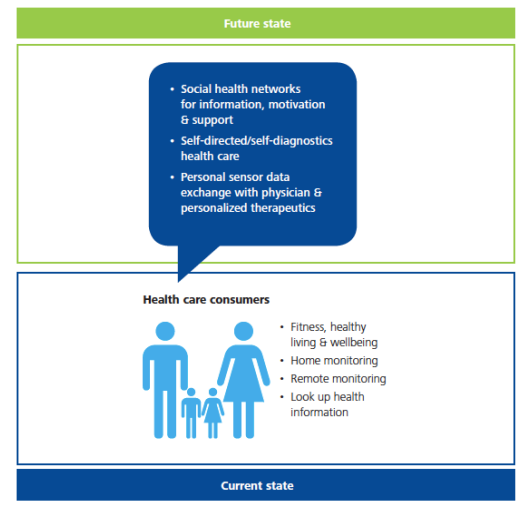

Fig. 1. Current and Future state of patient healthcare (adapted from [2 p.11])

The future of the patient experience is predicted to include social health networks for information access, support and motivation; self-directed and self-diagnostics capabilities for devices, personalised therapeutics and personal sensor data exchange with physicians. The future of healthcare will see the development of health capabilities with the capacity to capture and analyse self-reported data alongside objective behavioral and contextual information to extract meaning that positively impacts an individual's health status and well being [2]. This future vision provides a view on the nature of healthcare with the proliferation of PMDs in the near future. Similarly, [37] discusses the ways in which advanced wearable medical devices may impact the nature of healthcare. Patients interact via dedicated wearable technology as well as a variety of add-on gadgets functioning as sensors or data recorders continuously monitoring patient vital signs. The idea of traveling to a doctor's clinic will be an option of last resort as medical professionals and caregivers engage with patients remotely and provide health diagnosis based on data gathered from wearable devices. Patients will receive virtual and remote care in a variety of ways with virtual clinics providing urgent care through videoconferencing between patient and caregivers. For complicated cases wearable devices will be used to monitor specific conditions and alert emergency services while virtual procedures will become the norm as doctors will be able to use a variety of sensors to treat a patient without patients leaving the comfort of their own home. As we have identified it is likely that beyond wearable devices 
the forthcoming generation of devices will be more intimately integrated within the patients' bodies. Once PMDs move from the external to the internal a new dynamic begins to evolve. Internal devices verge on the concept of the 'cyborg' - a chimera of living organism and machine [9]. As identified earlier, implantable devices have the capacity to monitor, log and detect changes in the physical and physiological condition of the patient and control parts of the body autonomously and remotely. Although in their infancy, implantable device technologies are functioning at a high level of complexity with some devices designed to compensate for reduced sensory perception through to complicated technical systems capable of keeping living organisms alive [9].

Implantable devices present new experiences in the way patients manage and interact with healthcare. Instantaneous, immediate, automatic and involuntary interactions with the management of medical conditions could potentially occur anytime, anywhere without conscious thought from the patient. The implantable device itself would not include a physical interface and so traditional design issues regarding usability, functionality and ease-of-use would be irrelevant. For example consider a fully implantable smart diabetic monitor. A diabetes patient would go about their daily life while the device is constantly and automatically monitoring blood-glucose levels. Over time the device would determine when and how much insulin is required in the bloodstream depending on the food consumed and other factors. The device could upload this information automatically to an e-health system so that the patient's diet and information could be monitored to provide accurate longitudinal data for medical checkups, recommendations and future health plans. Relevant information about adjustments to diet and exercise could be delivered to the patient if a particular threshold was reached. In an emergency the implantable device could automatically alert emergency services and accurately pinpoint the location of the patient. It could also monitor the patient during this period and inform emergency personnel of any critical data that needs to be immediately addressed when they arrive for treatment. Another scenario could be a future implantable cardiac defibrillator device, early versions of which are currently available [8]. More advanced designs could have the capability to monitor heart rhythms and deliver small electrical shocks to adjust irregularities. Patients could go about their daily activities and the device would autonomously control irregular patterns in rhythms and even respond instantaneously to early signs of more severe heart problems and prevent the onset of a heart attack. Longitudinal data regarding could be monitored and logged to an external e-health network and analysed by a medical professional. Personal exercise and activity programs could be designed for the patient in response to the data history recorded. As technology progresses and PMDs begin to evolve opportunities exist for other scenarios. Devices implanted directly onto the brain could be used for treatment of conditions such as chronic pain, tremors and Parkinson's disease [9]. A range of biosensors used to measure biological phenomena and send data elsewhere for storage or analysis are also potential implantable systems [8]. Ingestible pills that enable inspection of cancerous growths in the intestinal wall and permit visual assessment of the overall health of the gastrointestinal tract are another form of implantable device [35]. All of these scenarios provide a window into the various ways in which PMDs have the potential to change the nature 
of healthcare. From a design perspective it is important to begin to consider how this shift impacts the experience of interaction and identify the ways design can be applied to positively contribute to these visions.

Impact on Patient Experience. As PMDs evolve in form, purpose and mode of use not only does the nature of healthcare transforms so too does the patient experience. From an experience design perspective how do these changes impact the patient experience? Once we move from external to wearable to internal, the design experience as we traditionally know it - transforms completely. Issues relating to tangible factors such as usability, physical button layout, interface design and so on almost disappear when it comes to internal devices. With devices that are internal it becomes all about the experience, or rather, what experience the device is programmed to deliver. As examined in the previous section fundamental aspects regarding the nature of healthcare changes as we move from external to wearable to internal (Section 5.1). Here we will propose ways in which the patient experience transforms through the lens of experience design. Table 1 illustrates the different ways that PMDs impact aspects of the patient experience. The top row outlines the modes of use as outlined previously including external, wearable and internal. For external type devices the interface that patients deal with are usually tangible and often include some sort of GUI. The device responds to the user input and as such can facilitate user intention or goal and mediate experience. Since the patient is driving the experience they are also aware of the interaction that is occurring and has a high degree of control. For wearable devices the interface is also tangible and may include a GUI but controls can sometimes be small or non-existent. Due to the nature of wearable devices, the device can respond to the user input but can also respond to the external context including environment, social setting, time of day, and so on; in other words wearable technology lends itself well to be context sensitive [38]. Wearable devices can mediate a users intention or goal but over time the interaction between can fade into the background, as there is only some degree of control on behalf of the patient. With implantable devices the experience between user and device changes in various ways. From a design perspective the biggest change is driven by the fact that implantable devices have no tangible interface since they are internal, but do have the potential to include an external GUI. One of the unique characteristics about implantable devices is that they not only have the capacity to respond to the patient's physiological, physical and internal conditions, they also have the capacity to control the patient and as a result not only mediate but also direct the experience. The device can continuously perform its function autonomously and so users can easily become unaware of its presence, as they are no longer controlling or managing the interaction. As healthcare merges with our internal physiological and physical body through devices that are implantable, intangible, and no longer consciously controlled by the user - what role will design play? These changes to patient interaction have clear and significant impacts on the nature of healthcare and the patient experience. The challenge is to identify the opportunity for design and to explore the ways design can engage effectively in this future scenario. 
Table 1. Portable medical device experience according to PMD changes

\begin{tabular}{|c|c|c|c|}
\hline Distance to body & External & Wearable & Internal \\
\hline Interface & $\begin{array}{l}\text { Tangible, graphical } \\
\text { user interface (GUI) }\end{array}$ & Tangible, GUI & Intangible, GUI \\
\hline $\begin{array}{l}\text { Device response condi- } \\
\text { tion }\end{array}$ & $\begin{array}{l}\text { Responds primarily to } \\
\text { patient input }\end{array}$ & $\begin{array}{l}\text { Responds to patient } \\
\text { input and context }\end{array}$ & $\begin{array}{l}\text { Respond to internal } \\
\text { condition of patient }\end{array}$ \\
\hline Experience potential & $\begin{array}{l}\text { Can mediate expe- } \\
\text { rience }\end{array}$ & $\begin{array}{l}\text { Can mediate expe- } \\
\text { rience }\end{array}$ & $\begin{array}{l}\text { Can mediate and } \\
\text { control experience }\end{array}$ \\
\hline Patient awareness & $\begin{array}{l}\text { Conscious device } \\
\text { interaction }\end{array}$ & $\begin{array}{l}\text { Interaction fades into } \\
\text { subconscious }\end{array}$ & $\begin{array}{l}\text { No longer aware of } \\
\text { device interaction }\end{array}$ \\
\hline Patient control & High degree & Some degree & Minimal to none \\
\hline
\end{tabular}

\section{Conclusion: A Dialogue on the Experience and Trends in Design of Portable Interactive Devices}

Devices that assist in patient healthcare should be designed to support the ongoing health and medical needs and overall quality of life for patients through effective user-device experience. PMDs play a critical role in healthcare that can lead to reduced pressure on the healthcare system, curtail unhealthy habits, increase the likelihood of improved lifestyle choices, enhance the user experience and ultimately raise the quality of life [2]. Designers need to be aware of the future trends in the PMD landscape and should apply experience design approaches to design effective, appropriate and positive user-product interactions and facilitate better healthcare and patient experiences $[17,18,19,23]$. This paper has identified some emerging trends in the design of PMDs; including changes in the form, purpose and mode of use, and explored how these trends are likely to impact the nature of healthcare and the patient experience from an experience design perspective. When PMDs evolve beyond wearable to implantable, and their mode of use changes from conscious to sub-conscious to no awareness of device interaction [39], patients will enter an era in which the very products themselves have the power to change the human condition [5]. Implantable devices will have the capacity to substantially impact physiology, behavior, and emotions as well as people's general health and wellbeing [2,5,9] ultimately rewiring the human brain. By addressing how people relate and interact with current and future PMDs, they can be better designed to suit the needs, requirements, wants and overall demands of patients within the healthcare system. Appropriately designed PMDs can permit patients to make informed decisions about their health and chronic conditions, take responsibility for their personal health management, motivate patients to make the right choice at the right time about their health, reduce the stress of managing chronic health, enhance experience and improve the overall quality of life for patients. From a design perspective there is a research opportunity to explore and better understand the ways these devices facilitate experiences for people in everyday life. Understanding the trends and the potential PMDs have to impact the nature of healthcare and the patient experience is a first step towards initiating a dialogue that will lead to effective and appropriate design strategies for PMDs in this new landscape. 


\section{References}

1. Kilian, J., Pantuso, B.: The Future of Health Care Is Social (2009), http: / /www. fastcompany.com/future-of-health-care

2. Greenspun, H., Coughlin, S.: mHealth in an mWorld: How mobile technology is transforming health care. Deloitte Center for Health Solutions, Washington (2012)

3. Geneau, R., Stuckler, D., Stachenko, S., McKee, M., Ebrahim, S., Basu, S., Chockalingham, A., Mwatsama, M., Jamal, R., Alwan, A., Beaglehole, R.: Raising the priority of preventing chronic diseases: A political process. Lancet 376, 1689-1698 (2010)

4. Hung, K., Zhang, Y.T., Tai, B.: Wearable Medical Devices forTele-Home Healthcare. In: 26th Annual International Conference on the IEEE EMBS, pp. 5384-5387 (2004)

5. Carr, N.: The shallows: What the Internet is doing to our brains. WW Norton \& Co. (2011)

6. Mirza, F., Norris, T., Stockdale, R.: Mobile technologies and the holistic management of chronic diseases. Hlth. Inf's. J. 14, 309-321 (2008)

7. Jones, M., Marsden, G.: Mobile interaction design. JohnWiley \& Sons, Chichester (2006)

8. Burleson, W., Clark, S., Ransford, B., Fu, K.: Design Challenges in Secure Implantable Medical Devices. In: ACM/IEEE DAC 2012 Conference, pp. 12-17 (2012)

9. Giselbrecht, S., Rapp, B., Niemeyer, C.: The Chemistry of Cyborgs-Interfacing Technical Devices with Organisms. Angew. Chem. Int. Ed. 52, 13942-13957 (2013)

10. The Economist: The Future of Healthcare in Europe (2011), http://www . janssen-emea.com/sites/default/files/

The-Future-Of-Healthcare-In-Europe.pdf

11. Chaytor, S., Staiger, U.: Future of Healthcare in Europe - Meeting future challenges: Key issues in context (2012), http://www.ucl.ac.uk/public-policy/public_ policy_publications / FHE-print.pdf

12. West, D.: Improving Health Care through Mobile Medical Devices and Sensors (2013), http: / /www. brookings . edu/ /media/research/files/papers / 2013 /

$10 / 22 \% 20$ mobile\%20medical\%20devices\%20west/

west_mobile\%20medical\%20devices_v06.pdf

13. Mechael, P.: The Case for mHealth in Developing Countries. Inn's: Tech, Gov's, Glob'n 4(1), 105-118 (2009)

14. Desmet, P., Hekkert, P.: Framework of product experience. Int. J. Des. 1, 57-66 (2007)

15. Overbeeke, C., Djajaningrat, J., Hummels, C., Wensveen, S.: Beauty in Usability: Forget about ease of use. In: Green, W., Jordan, P. (eds.) Pleasure with Products: Beyond Usability, pp. 9-18. Taylor and Francis, London (2002)

16. Schifferstein, H., Hekkert, P.: Product Experience. Elsevier, Amsterdam (2008)

17. Hassenzahl, M.: Experience Design: Technology for all the right reasons. Morgan \& Claypool, California (2010)

18. Gomez, R.: The evolving emotional experience with portable interactive devices. $\mathrm{PhD}$ thesis, Queensland University of Technology, Brisbane (2012)

19. Gomez, R., Popovic, V., Blackler, A.: A framework to better understand emotional experiences with portable interactive devices: Preliminary trial. In: 5th International Congress of IASDR, Tokyo (2013)

20. von Saucken, C., Michailidou, I., Lindemann, U.: How to Design Experiences: Macro UX versus Micro UX Approach. In: Marcus, A. (ed.) DUXU/HCII 2013, Part IV. LNCS, vol. 8015, pp. 130-139. Springer, Heidelberg (2013)

21. Mayne, T.: Emotions and health. In: Mayne, T., Bonanno, G. (eds.) Emotions: Current Issues and Future Directions, pp. 361-397. Guilford, New York (2001) 
22. Gloyd, D.: Positive user experience and medical adherence. In: International Conference on DPPI, pp. 17-21. ACM Publications, New York (2003)

23. Gomez, R., Popovic, V., Blackler, A.: Emotional experience with portable health devices. In: 7th International Conference on D\&E, Chicago (2010)

24. Fairbanks, R., Wears, R.: Hazards with medical devices: the role of design. Ann. of Emerg. Med. 52, 519-521 (2008)

25. Sharples, S., Martin, J., Lang, A., Craven, M., O'Neill, S., Barnett, J.: Medical Device Design in Context: A Model of User-device Interaction and Consequences. Displays 33(4-5), 221-232 (2012)

26. Ahmadvand, A., Fayaz-Bakhsh, A.: Mobile Devices and Applications for Health: An Exploratory Review of the Current Evidence with Public Health Perspective. Ira'n. J. of Med'l. Inf's. 1(2), 6-14 (2012)

27. Glaros, C., Fotiadis, D.: Wearable Devices in Healthcare. In: Hutchison, D., Shepherd, W.D., Mariani, J.A. (eds.) Intelligent Paradigms for Healthcare Enterprises. STUDFUZZ, vol. 184, pp. 237-264. Springer, Heidelberg (2005)

28. McCurdie, T., Taneva, S., Casselman, M., Yeung, M., McDaniel, C., Ho, W., Cafazzo, J.: mHealth Consumer Apps: The Case for User-Centered Design. Biomed. Instrum. Tech. 46, 49-56 (2012)

29. Dolan, B.: 7 medical phone peripherals you should know, (2011),

http: / /mobihealthnews. com/12062/

7-medical-phone-peripherals-you-should-know/6/

30. Bai, J., Zhang, Y., Shen, D., Wen, L., Ding, C., Cui, Z., Tian, F., Yu, B., Dai, B., Zhang, J.: A portable ECG and blood pressure telemonitoringsystem. IEEE Engineering in Medicine and Biology Magazine 18(4), 63-70 (1999)

31. Anliker, U., Ward, J., Lukowicz, P., Tröster, G., Dolveck, F., Baer, M., Keita, F., Schenker, E., Catarsi, F., Coluccini, L., Belardinelli, A., Shklarski, D., Alon, M., Hirt, E., Vuskovic, M.: AMON: A wearable multiparameter medical monitoring and alert system. IEEE Trans. Inform.Technol. Biomed. 8(4), 415-427 (2004)

32. Consolvo, S., McDonald, D., Landay, J.: Theory-driven design strategies for technologies that support behavior change in everyday life. In: CHI 2009, pp. 405-414 (2009)

33. Byrne, R., Eslambolchilar, P.: Encouraging an Active Lifestyle with Personal MobileDevices: Motivational Tools and Techniques. In: First International Workshop on Nudge \& Influence Through Mobile Devices, MobileHCI 2010 (2010)

34. Chan, M., Estve, D., Fourniols, J., Escriba, C., Campo, E.: Smartwearable systems: Current status and future challenges. Art. Intl. in Med. 56(3), 137-156 (2012)

35. Chen, I.-M., Phee, S., Luo, Z.: Personalized biomedical devices \& systems for healthcare applications. Front's. of Mech. Eng. 6(1), 3-12 (2011)

36. Wallace, G., Higgins, M., Moulton, S., Wang, C.: Nanobionics: The impact of nanotechnology on implantablemedical bionic devices. Nanoscale 4, 4327-4347 (2012)

37. Schroetter, J.: The Future of Wearable Computing in Healthcare (2014), http: / / www . matmag.com/blogs / 2014/01/future-wearablecomputing-healthcare

38. Lukowicz, T., Kirstein, G., Troster, G.: Wearable systems for health care applications. Meth. Inf. Med. 43, 232-238 (2004)

39. Lookout.: Mobile Mindset Study (2012), https: //www. lookout.com/resources/reports/mobile-mindset 